\title{
SIMULTANEOUS INTEGRAL/RXTE OBSERVATIONS OF GRS 1915+105
}

\author{
J. Rodriguez, ${ }^{1,2}$ D. Hannikainen, ${ }^{3}$ O. Vilhu, ${ }^{3,2}$ Y. Fuchs, ${ }^{1}$ and S. E. Shlaw ${ }^{1.2}$ \\ RESUMEN
}

Presentamos los primeros resultados de observaciones INTEGRAL y RXTE simultáneas del mic(ucuasar GRS 1915+105. Nos enfocamos en el análisis de la única observación altamente variahle. mostrando que pudimos haber observado una nueva clase de variabilidad. Luego estudiamos la dependencia chergćtica de una QPO con base en nuestras observaciones regulares.

\section{ABSTRACT}

We present the first results of simultaneous INTEGRAL and RXTE observations of the microquasar GRS $1915+105$. We focus on the analysis of the unique highly variable observation and show that we might have observed a new class of variability. We then study the energetic dependener of a low frechurner (QPO from our steady observations.

Key Words: STARS: BINARIES - STARS: INDIVIDUAL (GRS1915+105) — X-RAYS: BURSTS

1. GENERALITIES ON GRS $1915+105$

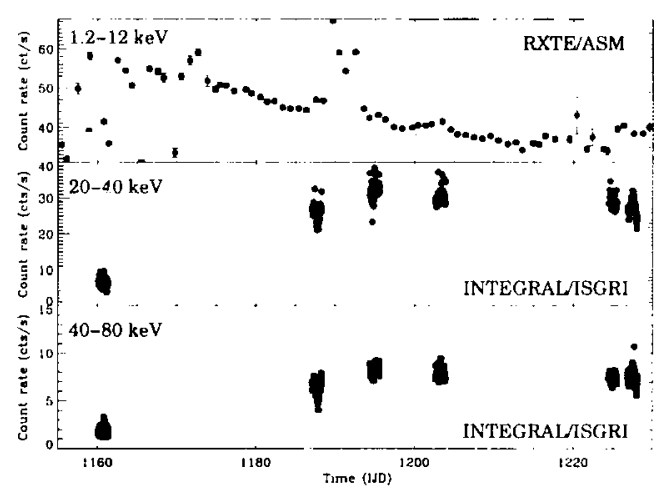

Fig. 1. RXTE/ASM (top) and INTEGRAL/ISGRI (middle ad bottom) light curves during spring 2003.

Since its discovery in 1992 (Castro-Tirado et al. 1992), the study of GRS $1915+105$ at all wavelengths has given us a unique insight into the accretionejection coupling in microquasars. While the almost daily coverage with RXTE allowed to classify the X-ray variability into 12 classes (Belloni et al. 2000), multiwavelength coverage showed a correlation between radio ejections and X-ray variability (e.g. Mirabel et al. 1998). GRS 1915+105, is also one of the 2 microquasars for which a compact jet has been imaged using radio interferometry (Dhawan et al. 2000, Fuchs et al. 2003).

GRS $1915+105$ was observed with INTEGRAL during 6 observations of at least $100 \mathrm{ks}$ (revolution

\footnotetext{
${ }^{1}$ CEA/Service d'Astrophysique, Saclay, France.

${ }^{2}$ Integral Science Data Center, Versoix, Switzerland.

${ }^{3}$ University of Helsinki, Helsinki, Finland.

${ }^{4}$ University of Southampton, Southampton, UK.
}

$48,59,69,70+57 \& 62$ discussed in Fuchs et al. 2003 and these proceedings). Sinnultaneous R RTE observations were performed cluring 4 of these 6 observations. Fig.1 shows the RXTE/A.SM daily light curve and and the INTEGRAL/ISGRI light curver over the 6 observations.

\section{FIRST INTEGRAL OBSERVATION OF GRS $1915+105$}

The first striking behavior of this first olsservittion is the recurrence of large spikes on tims siale $\sim$; min (Fig. 2). The production of a power spectrum showed a strong QPO at $3 \mathrm{mHz}$, indicating that the observation is dominated by these carliat ions (Hanlnikainen et al. 2003). This light (mve resombles the

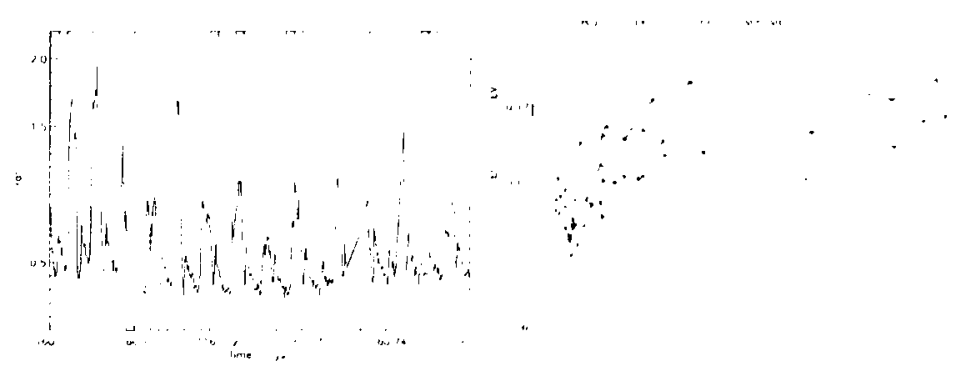

Fig. 2. Left: Zoom on the JEXI-X lighteurve during rex, 48. Right: Mran hardness ratio vs. count ratte for 300 spikes as seen by JFAl-X

$\rho$ class of Belloni ot al. (2000). howerver the time arale is different. A more precise study allowed us to further distinguish it from any chass of valuability known up to now. Indered when we sully the hatreduess ratio of the sonder. it apperats that the rising part of the spikes is hard and the decaly is solt. This is nut texperterl if we suppose the rising part of the spikes is 
due to a disk instability, and the decay represents the receding of the disk. This kind of behavior could be evidence for the presence of two independent accretion flows, obeying different time scales. In that case the disk would react to any perturbation of the accretion rate on a viscous time scale, while the corona would react on a free fall time scale. Such a conclusion has been drawn in the case of other black hole binaries (with low mass stars), e.g. 1E 1740.7-2942, GX 339-4, GRS 1758-258 (Smith et al. 2002) or XTE J1550-564 (Rodriguez et al. 2003). The recurrence time of the peaks in GRS $1915+105$ is not comparable with any of these black hole binaries, however. More precise studies of this class, e.g. time resolved spectroscopy and comparison between the dips, spikes, rising and decaying phases of the peaks should allow us to obtain more information on the physics underlying these phenomena.

\section{STEADY STATE OBSERVATIONS: QPO STUDIES}

GRS $1915+105$ was observed in a steady state during all the other observations. A typical example of RXTE/INTEGRAL spectral analysis is presented in Fuchs et al. (2003, and these proceedings). During all the observations for which we had a simultaneous RXTE coverage, the spectral parameters are comparable. In all observations a strong $(\sim 14 \%$ rms amplitude) low frequency quasi-periodic oscillation (LFQPO) is clearly detected (e.g. Fuchs et al. 2003). Given the long RXTE exposure times and the high luminosity of the source we could study the energetic dependence of the LFQPO parameters with the highest possible spectral resolution. Both the frequency and the width do not depend on the energy. The evolution of the amplitude vs. the frequency is plotted on Fig. 3 for rev 57,59 and 69. A

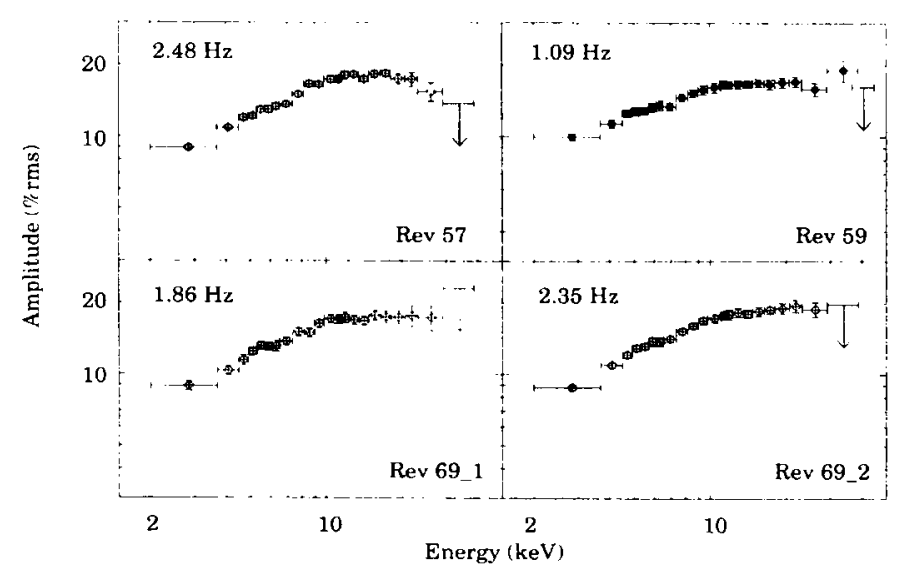

Fig. 3. Energy dependence of the LFQPO detected during the 4 observations labeled in each panel. The frequency of the feature is also indicated. cut-off around $15 \mathrm{keV}$ is clearly detected in rev. 57 , confirming previous findings (e.g. Rodriguez et al. 2002). The position of the cut-off might be simply shifted towards high energies in the other observations. The statistics of the PCA data above $25 \mathrm{keV}$ prevents, however, any firm conclusions. The origin of the cut-off is still unclear, and might indicate different phenomena: either the QPO moves relative to the corona (see e.g. Tomsick \& Kaaret 2001), or the shape of the QPO spectrum might be evidence for a hot spot rotating in the disk (Rodriguez et al. 2002). In a simpler manner, if a significant part of the high energy photons is emitted through synchrotron radiation by a compact jet (which is present at least during rev 57; Fuchs et al. 2003), then the shape of the QPO spectra can be easily understood if one assumes the QPO does not originate in the jet. Once again this would point toward a strong coupling of the QPO with the Compton corona as usually observed, and in agreement with most models of LFQPOs (e.g. Accretion Ejection Instability, or CENtrifugal Dominated BOundary Layer). In the future the use of INTEGRAL will help to better constrain the spectra of QPO at high energy, and probe their relation to the spectral parameters while performing wide band spectroscopy.

The authors thank T. Belloni, M. Tagger, P. Varnière, G. Henry, P.-O. Petrucci, C. Cabanac, S. Chaty, M. Ribo, N.J. Westergaard for useful discussions and comments on the present work. JR and YF acknowledge financial support from the CNES. DH is a Research Fellow of the Finnish Academy.

\section{REFERENCES}

Belloni, T., Klein-Wolt, M., Méndez, M. et al. 2000, A\&A, 355, 271

Castro-Tirado, A.J., Brandt, S., Lund, N. 1992, IAUC 5590

Dhawan, V., Mirabel, I.F., Rodríguez, L.F. 2000, ApJ, 543,373

Fuchs, Y., Rodriguez, J., Mirabel, I.F., et al. 2003, A\&A, 409, L.35

Hannikainen, D.C., Vilhu, O., Rodriguez, J., et al. 2003, A\&A, 411, L.415

Mirabel, I.F., Dhawan, V., Chaty, S. et al. 1998, A\&A, 330, L9

Rodriguez, J., Durouchoux, P., Mirabel, I.F. et al. 2002, A\&A, 386, 271

Rodriguez, I., Corbel, S., Tomsick, J.A. 2003, ApJ, 595, 1032

Smith, D.M., Heindl, W.A., Swank, J.H. 2002, ApJ, 560, 362

Tomsick, J.A., Kaaret, P. 2001, ApJ, 548, 401 